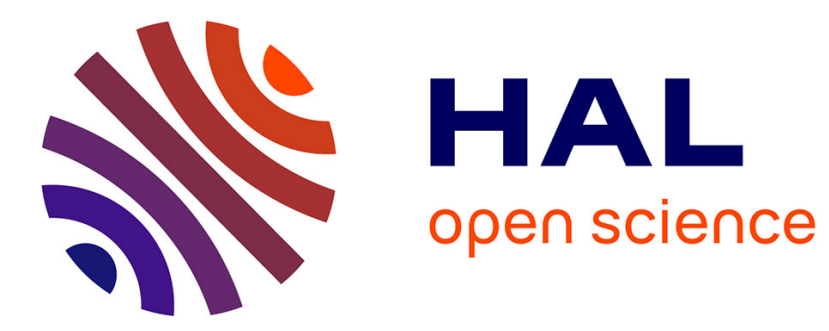

\title{
Application of the spectral element method to the solution of the multichannel Schroedinger equation
}

\author{
Andrea Simoni, Alexandra Viel, Jean-Michel Launay
}

\section{To cite this version:}

Andrea Simoni, Alexandra Viel, Jean-Michel Launay. Application of the spectral element method to the solution of the multichannel Schroedinger equation. Journal of Chemical Physics, 2017, 146 (24), pp.244106-1-9. 10.1063/1.4987026 . hal-01551686

\section{HAL Id: hal-01551686 \\ https://hal.science/hal-01551686}

Submitted on 30 Jun 2017

HAL is a multi-disciplinary open access archive for the deposit and dissemination of scientific research documents, whether they are published or not. The documents may come from teaching and research institutions in France or abroad, or from public or private research centers.
L'archive ouverte pluridisciplinaire HAL, est destinée au dépôt et à la diffusion de documents scientifiques de niveau recherche, publiés ou non, émanant des établissements d'enseignement et de recherche français ou étrangers, des laboratoires publics ou privés. 
Application of the spectral element method to the solution of the multichannel Schrödinger equation

Andrea Simoni, Alexandra Viel, and Jean-Michel Launay

Citation: The Journal of Chemical Physics 146, 244106 (2017); doi: 10.1063/1.4987026

View online: http://dx.doi.org/10.1063/1.4987026

View Table of Contents: http://aip.scitation.org/toc/jcp/146/24

Published by the American Institute of Physics

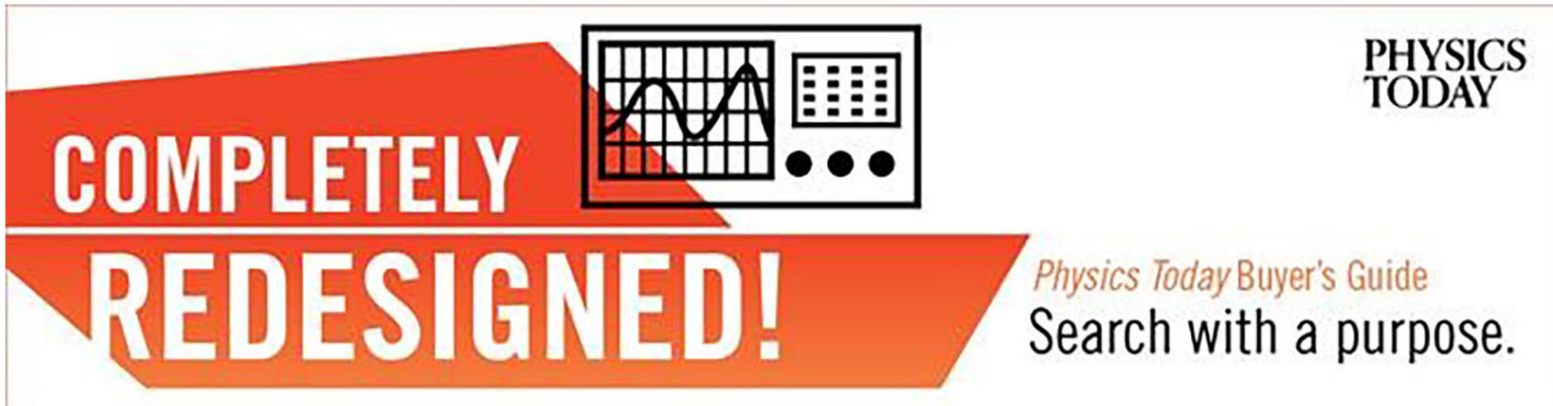




\title{
Application of the spectral element method to the solution of the multichannel Schrödinger equation
}

\author{
Andrea Simoni, Alexandra Viel, and Jean-Michel Launay \\ Institut de Physique de Rennes, UMR 6251, CNRS and Université de Rennes 1, 35042 Rennes Cedex, France
}

(Received 7 February 2017; accepted 8 June 2017; published online 26 June 2017)

\begin{abstract}
We apply the spectral element method to the determination of scattering and bound states of the multichannel Schrödinger equation. In our approach, the reaction coordinate is discretized on a grid of points whereas the internal coordinates are described by either purely diabatic or locally diabatic (diabatic-by-sector) bases. Bound levels and scattering matrix elements are determined with spectral accuracy using relatively small number of points. The scattering problem is cast as a linear system solved using state-of-the-art sparse matrix non-iterative packages. Boundary conditions can be imposed so as to compute a single column of the matrix solution. A comparison with log-derivative propagators customarily used in molecular physics is performed. The same discretization scheme can also be applied to bound levels that are computed using direct scalable sparse-matrix solvers. Published by AIP Publishing. [http://dx.doi.org/10.1063/1.4987026]
\end{abstract}

\section{INTRODUCTION}

The solution of partial differential equations, ubiquitous in all areas of physics, can be tackled by a variety of numerical methods developed over the last decades. Solution algorithms can essentially be divided into propagation and basis expansion approaches. The former build the solution iteratively from a known initial value up to the final propagation distance, where suitable boundary conditions are imposed. Such methods are easy to implement and cheap in memory storage but provide relatively low convergence rates as a function of the step size. Large number of steps may therefore be needed, such that accumulation of round-off errors can limit the accuracy, in particular, for complex systems. Due to its pivotal role in quantum dynamics, the time-independent Schrödinger equations have been granted particular attention in the molecular physics community. Popular propagation algorithms include the log-derivative propagator of Johnson, ${ }^{1}$ later improved by Manolopoulos, ${ }^{2}$ and the renormalized Numerov algorithm. ${ }^{3}$

In basis expansions, the solution is determined altogether as a development over a basis, usually trigonometric or polynomial, with suitable conditions imposed at the boundaries. A main advantage is the exponential numerical convergence as a function of the expansion order. ${ }^{4}$ Grid-discretization methods are particular basis expansions in which each basis function is nonzero at a unique grid point. In global approaches, the whole interval of interest is represented as a discrete grid of points. Global grid techniques have been introduced in molecular physics in the context of the so-called discrete variable representation (DVR). ${ }^{5}$ One drawback of global grids is the need to introduce nonlinear coordinate transformations to efficiently represent complex solutions varying on disparate length scales such as ultracold processes. ${ }^{6,7}$

Local approaches subdivide the interval of interest in subintervals, often termed as elements. Basis functions localized in subintervals are used to expand the solution. As compared to global approaches, since the resulting matrix is sparse one can apply performant sparse linear algebra packages to carry out the operations needed in the specific problem. Moreover, at least in one-dimensional problems, the element size can be tailored to the solution in a straightforward way.

One widespread local approach is the finite element method. ${ }^{8}$ Being based on low order polynomial expansions, such a method requires a large number of points to achieve high accuracy. Use of high order polynomials as basis functions in each element marked the birth of spectral element approaches few decades ago. ${ }^{9}$ The spectral element method is nowadays a well established tool in scientific and engineering computations. ${ }^{10,11}$

Use of this computational technique in molecular physics has been pioneered for one-dimensional problems in Ref. 12 . More recently, a spectral element approach in two spatial dimensions has been presented in Refs. 13 and 14, where it was termed as finite-element DVR. Appropriate scattering boundary conditions were imposed using a spatial rotation in the complex plane known as the exterior complex scaling approach. ${ }^{13,15}$ A combination of the spectral element and the slow variable representation ${ }^{16}$ has been proposed in order to compute weakly bound states of triatomic systems in the hyperspherical framework. ${ }^{17}$ Finally, the finite-element DVR has been used as a time-independent representation in multidimensional time-dependent calculations; see, e.g., Refs. 18-20.

The main aim of the present work is to explore the usefulness of the spectral element method in quantum dynamics for time-independent multichannel problems. More particularly, we point out that combining the spectral element method with traditional molecular basis or diabatic-by-sector expansions $^{21}$ to treat the internal coordinates optimizes sparsity and size of the discretized Hamiltonian. One major advantage is that the wavefunction is obtained at no extra computational cost. Moreover, the spectral nature of the method allows the 
accuracy of the solution to be estimated reliably in each region of space. Subsequent step refinements lead to a grid tailored to the interaction strength in various regions of space. We show that the spectral element formulation lends itself to imposing in a natural way different boundary conditions for scattering and bound-state calculations.

The paper is organized as follows: Sec. II presents the discretization scheme and introduces various boundary conditions, Sec. III discusses numerical results on a realistic rovibrational system, and Sec. IV summarizes and concludes this work.

\section{HAMILTONIAN DISCRETIZATION}

Fundamentals of the spectral-element approach can be found in textbooks and articles. ${ }^{11-13}$ In order to set the notation and illustrate the specific approach we follow to combine full or locally diabatic expansions and the grid basis, we reproduce in this section the main steps of the derivation from scratch.

We consider a generic time-independent multidimensional problem and identify an "external" reaction coordinate $R$ describing the "size" of the system and a set of "internal" variables denoted collectively as $\Omega$. For instance, in the twobody problem, $R$ typically represents the distance between the particles and $\Omega$ represents the orientation of the inter-particle position vector. In three-body systems, $R$ may represent the hyperradius and $\Omega$ may represent a set of hyperangles. Note that in general $\Omega$ may comprise coordinates with physical dimension of length, like in the case of our test atom-molecule ro-vibrational problem described in detail in Sec. III.

The time-independent Schrödinger equation is schematically written as

$$
\left[-\frac{\hbar^{2}}{2 \mu} \frac{\partial^{2}}{\partial R^{2}}+V(R, \Omega)\right] \Psi(R, \Omega)=E \Psi(R, \Omega)
$$

which is to be solved in the hyper-region $R_{\min } \leq R \leq R_{\max }$. Here $V$ contains various potential energy terms and/or differential operators acting on the internal variables $\Omega$. The derivation turns out to be formally simpler if, in the spirit of the slow variable representation, ${ }^{16} R$ is discretized first and the internal variables $\Omega$ at a second stage.

The radial interval is partitioned into $M$ subintervals or elements. We generate in each subinterval $m$ a set of $P_{m}$ Gauss-Lobatto points and weights $\left(R_{p}^{(m)}, w_{p}^{(m)}\right), p=1, \ldots, P_{m}$, with $R_{1}^{(m)}$ and $R_{P_{m}}^{(m)}$ the subinterval endpoints. ${ }^{4}$ Note that since the endpoints of contiguous intervals are such that $R_{P_{m-1}}^{(m-1)}=R_{1}^{(m)}$, the number of distinct points in the complete grid is $L=\sum_{m=1}^{M}\left(P_{m}-1\right)+1$. The local points and weights can be used to implement the Gauss-Lobatto quadrature

$$
\int_{R_{1}^{(m)}}^{R_{P_{m}}^{(m)}} f(R) d R=\sum_{p=1}^{P_{m}} w_{p}^{(m)} f\left(R_{p}^{(m)}\right),
$$

an integration rule exact for polynomials up to degree $2 P_{m}$ -3 . Each point can be associated with a Gauss-Lobatto cardinal or shape function defined such that $C_{i}^{(m)}\left(R_{p}^{(m)}\right)=\delta_{i p}$ at the nodal points inside the element and continued as identically zero outside the element, $C_{i}^{(m)}(R)=0$, if $R \notin\left[R_{1}^{(m)}, R_{P_{m}}^{(m)}\right]$. The $C_{i}^{(m)}$ functions can be obtained by linear mapping in terms of the corresponding cardinal functions $c_{i}(x)$ of the primitive interval $x \in[-1,1]$,

$$
C_{i}^{(m)}(R)=c_{i}\left(2 \frac{R-R_{1}^{(m)}}{R_{P_{m}}^{(m)}-R_{1}^{(m)}}-1\right) .
$$

Explicitly, $c_{i}$ for $N$ grid points can be expressed in terms of the derivatives of the Legendre polynomial of order $N-1$ as follows: 4

$$
c_{i}(x)=\frac{-\left(1-x^{2}\right)}{N(N-1) P_{N-1}\left(x_{i}\right)\left(x-x_{i}\right)} \frac{d P_{N-1}(x)}{d x},
$$

where the Gauss-Lobatto points $x_{i}$ in the primitive interval comprise the endpoints \pm 1 and the $(N-2)$ zeros of the $\frac{d P_{N-1}(x)}{d x}$ polynomial.

The Gauss-Lobatto cardinal functions associated with the internal points $p=2, \ldots, P_{m}-1$ vanish at the element endpoints $R=R_{1}^{(m)}, R_{P_{m}}^{(m)}$ and following Ref. 11 will be referred to as "internal functions." We also conventionally consider as internal the cardinal functions relative to the first $R_{1}^{(1)}$ and last $R_{P_{M}}^{(M)}$ grid points. For each internal point, that is, for $m=2, \ldots, M-1$ and $i=2, \ldots, P_{m}-1$ as well as for $(i$, $m)=(1,1)$ and $(i, m)=\left(P_{M}, M\right)$, we will simply take as basis functions the cardinal functions

$$
\mathcal{C}_{i}^{(m)}(R)=C_{i}^{(m)}(R) .
$$

The construction of the basis functions associated with the remaining $(M-1)$ inter-element points $R_{P_{m-1}}^{(m-1)}=R_{1}^{(m)}$ with $m=2, \ldots, M$ is obtained by "glueing" cardinal functions. ${ }^{11}$ These interface or bridge functions are defined by

$$
\mathcal{C}_{1}^{(m)}(R)=\left\{\begin{array}{l}
C_{P_{m-1}}^{(m-1)}(R), R \in\left[R_{1}^{(m-1)}, R_{P_{m-1}}^{(m-1)}\right], \\
C_{1}^{(m)}(R), \quad R \in\left[R_{1}^{(m)}, R_{P_{m}}^{(m)}\right],
\end{array}\right.
$$

for $m=2, \ldots, M$. Note that contiguous subintervals are only connected through such interface functions.

In order to build a global representation of the Hamiltonian, we now introduce a single index $a=1, \ldots, L$ running over the $L$ distinct points of the full grid and note $R_{a}$ such distinct grid points. We define global weights

$$
\omega_{a}= \begin{cases}\left(w_{P_{m-1}}^{(m-1)}+w_{1}^{(m)}\right) & , \text { if } R_{a} \text { is inter-element } \\ w_{p}^{(m)}, & \text { otherwise. }\end{cases}
$$

Similarly, we build a global grid basis comprising internal and interface functions

$$
\mathcal{C}_{a}(R)=\left\{\begin{array}{l}
\mathcal{C}_{1}^{(m)}, \text { if } R_{a} \text { is inter-element } \\
\mathcal{C}_{p}^{(m)}, \text { otherwise }
\end{array}\right.
$$

Equations (5) and (8) guarantee that the orthogonality relation,

$$
\int_{R_{1}}^{R_{L}} \mathcal{C}_{b}(R) \mathcal{C}_{a}(R) d R=\delta_{b a} \omega_{a}
$$

holds with at least Gaussian quadrature accuracy; see Eq. (2).

For each value of the internal coordinates $\Omega$, we now develop the system wavefunction on the radial basis

$$
\Psi(R, \Omega)=\sum_{a=1}^{L} \Phi_{a}(\Omega) \mathcal{C}_{a}(R),
$$


the coefficients being equal to the wavefunction evaluated at the grid points $\Phi_{a}(\Omega)=\Psi\left(R_{a}, \Omega\right)$. The Schrödinger equation is now projected on the basis functions $\mathcal{C}_{a}$. The second derivative arising from the radial kinetic energy term gives rise to an integral in $R$ that is further developed as a sum of integrals restricted to each element,

$$
\begin{aligned}
\int_{R_{1}}^{R_{L}} \mathcal{C}_{a}(R) \frac{\partial^{2} \Psi(R, \Omega)}{\partial R^{2}} d R= & \sum_{m=1}^{M} \int_{R_{1}^{(m)}}^{R_{P_{m}}^{(m)}} \mathcal{C}_{a}(R) \frac{\partial^{2} \Psi(R, \Omega)}{\partial R^{2}} d R \\
= & \sum_{m=1}^{M}\left(-\int_{R_{1}^{(m)}}^{R_{P_{m}}^{(m)}} \frac{\partial \mathcal{C}_{a}(R)}{\partial R} \frac{\partial \Psi(R, \Omega)}{\partial R} d R\right. \\
& \left.+\left[\mathcal{C}_{a}(R) \frac{\partial \Psi(R, \Omega)}{\partial R}\right]_{R_{1}^{(m)}}^{R_{P_{m}}^{(m)}}\right),
\end{aligned}
$$

where one integration by parts has been performed for the second equality. Noticing that for two consecutive elements one has

$$
\mathcal{C}_{a}\left(R_{P_{m}}^{(m)}\right) \frac{\partial \Psi\left(R_{P_{m}}^{(m)}, \Omega\right)}{\partial R}=\mathcal{C}_{a}\left(R_{1}^{(m+1)}\right) \frac{\partial \Psi\left(R_{1}^{(m+1)}, \Omega\right)}{\partial R}
$$

Equation (11) reduces to

$$
\begin{aligned}
\int_{R_{1}}^{R_{L}} \mathcal{C}_{a}(R) \frac{\partial^{2} \Psi(R, \Omega)}{\partial R^{2}} d R= & -\sum_{m=1}^{M} \int_{R_{1}^{(m)}}^{R_{P_{m}}^{(m)}} \frac{\partial \mathcal{C}_{a}(R)}{\partial R} \frac{\partial \Psi(R, \Omega)}{\partial R} d R \\
& +\mathcal{C}_{a}\left(R_{L}\right) \frac{\partial \Psi\left(R_{L}, \Omega\right)}{\partial R} \\
& -\mathcal{C}_{a}\left(R_{1}\right) \frac{\partial \Psi\left(R_{1}, \Omega\right)}{\partial R} .
\end{aligned}
$$

The boundary terms' cancellation of Eq. (12) holds for the exact solution but is only approximately valid when the solution is computed as an expansion on a finite basis. In other terms, the numerical solution in general will have a discontinuous derivative at the element interfaces. In spite of such discontinuity, it can be proved that the numerical error tends to zero exponentially with the number of basis functions when expressed in the energy norm, which measures the error both in the function value and derivative; see, e.g., Ref. 11. Note that convergence in the energy norm does not imply uniform or even point-wise convergence. However, although we are not aware of a formal proof, in our tests performed in Sec. III, we numerically find that the discontinuity in the derivative tends to zero exponentially not affecting the fast convergence rate in the quantities of interest.

Using the decomposition Eq. (10) for the evaluation of $\partial \Psi(R, \Omega) / \partial R$, one gets a term involving the matrix

$$
\mathcal{T}_{a b}=\sum_{m=1}^{M} \int_{R_{1}^{(m)}}^{R_{P_{m}}^{(m)}} \frac{d \mathcal{C}_{a}(R)}{d R} \frac{d \mathcal{C}_{b}(R)}{d R} d R
$$

formally recast as

$$
\mathcal{T}_{a b}=\int_{R_{1}}^{R_{L}} \frac{d \mathcal{C}_{a}(R)}{d R} \frac{d \mathcal{C}_{b}(R)}{d R} d R .
$$

Using the definitions Eqs. (5) and (6), the linear mapping in Eq. (3), and approximating the integrals on the rhs by the quadrature of Eq. (2), the kinetic matrix can be expressed in terms of analytically known Gauss-Lobatto derivation matrices $C_{j}^{\prime}\left(x_{i}\right)$; see, e.g., Ref. 4 .
A closer look at Eq. (14) keeping into account the local character of the $\mathcal{C}_{a}(R)$ functions shows that most elements of $\mathcal{T}$ are zero. More specifically, $\mathcal{T}_{a b}=0$ if $\mathcal{C}_{a}$ and $\mathcal{C}_{b}$ are both internal functions and do not belong to the same element. If $\mathcal{C}_{a}$ is an interface function, thus at the interface of two elements $m$ and $m+1, \mathcal{T}_{a b}=0$ if $b$ does not belong to any of the two $m$ and $m+1$ elements, while $\mathcal{T}_{a b} \neq 0$ if $\mathcal{C}_{b}$ is an internal or an interface function of element $m$ or $m+1$. In addition, $\mathcal{T}_{a b} \neq 0$ if both $a$ and $b$ belong to the same element. The potential energy is approximately diagonal in the grid basis with diagonal elements $\omega_{a} V\left(R_{a}, \Omega\right)$ if quadrature Eq. (2) is used. Collecting all the terms, the matrix form of the Schrödinger equation finally reads

$$
\begin{gathered}
\sum_{b=1}^{L} \mathcal{T}_{a b} \Phi_{b}(\Omega)+\frac{2 \mu}{\hbar^{2}} \omega_{a}\left[V\left(R_{a}, \Omega\right)-E\right] \Phi_{a}(\Omega) \\
=\left.\delta_{L a} \partial_{R} \Psi\right|_{R=R_{L}}-\left.\delta_{1 a} \partial_{R} \Psi\right|_{R=R_{1}} .
\end{gathered}
$$

We now introduce an internal coordinate basis $\phi_{\alpha}(\Omega)$ whose nature or dimension is not needed for the moment to be specified. We write therefore

$$
\Phi_{a}(\Omega)=\sum_{\alpha=1}^{N^{(a)}} F_{a \alpha} \phi_{\alpha}^{(a)}(\Omega),
$$

where the superscript $(a)$ stresses the possible dependence of the basis on the grid point. Insertion of Eq. (17) in Eq. (16) leads to the algebraic equation,

$$
\begin{aligned}
& \sum_{b=1}^{L} \sum_{\beta=1}^{N^{(b)}} \mathcal{T}_{a b} \mathcal{O}_{a \alpha, b \beta} F_{b \beta}+\frac{2 \mu}{\hbar^{2}} \sum_{\beta=1}^{N^{(a)}} \omega_{a}\left[\mathcal{U}_{\alpha \beta}\left(R_{a}\right)-E \delta_{\alpha \beta}\right] F_{a \beta} \\
& \quad=\delta_{L a} \xi_{\alpha L}-\delta_{1 a} \xi_{\alpha 1}
\end{aligned}
$$

where $\mathcal{O}_{a \alpha, b \beta}=\left\langle\phi_{\alpha}^{(a)} \mid \phi_{\beta}^{(b)}\right\rangle_{\Omega}$ is the overlap matrix element over the $\Omega$ coordinates and $\mathcal{U}_{\alpha \beta}\left(R_{a}\right)=\left\langle\Phi_{\alpha}^{(a)}(\Omega)\left|V\left(R_{a}, \Omega\right)\right| \Phi_{\beta}^{(a)}(\Omega)\right\rangle$. The quantities $\xi_{\alpha a}$ with $a=1, L$ are the normal derivatives of the wavefunction at the integration boundaries in channel $\alpha$, i.e.,

$$
\xi_{a \alpha}=\left\langle\phi_{\alpha}^{(a)}\left|\partial_{R} \Psi\right|_{R=R_{a}}\right\rangle_{\Omega} .
$$

Equation (18), supplemented by the proper boundary conditions in Secs. II A and II B, represents the key formal result of the paper. In order to maximize sparsity, one requires that the basis $\phi_{\alpha}^{(a)}$ does not depend on the grid point $a$. This is, for example, the case when using spherical or hyperspherical harmonics, or ro-vibrational molecular states. In this case, the $\mathcal{O}$ matrix reduces to the identity matrix.

A pictorial representation of the resulting Hamiltonian matrix is given in Fig. 1 where each small block corresponds to fixed grid indices and varying channel indices. Similar matrix structure representations can be found elsewhere in the literature, for instance, in Refs. 15 and 20. For this example, three subintervals $M=3$ are considered; the number of Gauss-Lobatto points and the number of basis for the internal coordinates are identical for the three subelements and are fixed to $P_{m}=4$ and $N^{(a)}=5$ channels. The non-zero elements arising from the kinetic part are depicted in gray while the ones resulting from the potential are in blue. For the illustrative case presented, 340 over the $50 \times 50$ matrix elements are non-zero, which amounts to a filling factor of $13.6 \%$. 


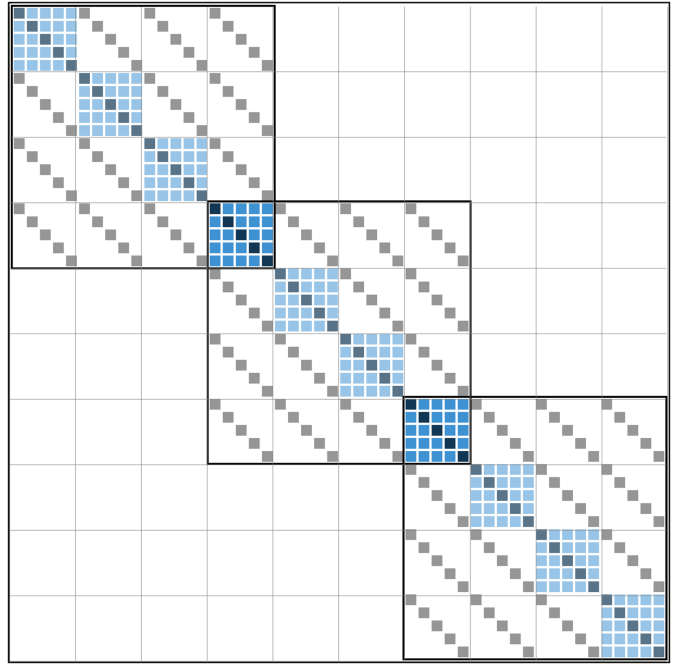

FIG. 1. Structure of the discretized matrix for 3 elements, 4 Lobatto points, and 5 channels per element. Each full block along the main diagonal (blue) contains the 25 channel matrix elements $\mathcal{T}_{a a} \delta_{\alpha \beta}+\frac{2 \mu}{\hbar^{2}} \omega_{a}\left[\mathcal{U}_{\alpha \beta}\left(R_{a}\right)-E \delta_{\alpha \beta}\right]$ for $\alpha, \beta=1, \ldots, 5$ on the lhs of the equation system Eq. (18). Each offdiagonal diagonal block (gray) only arises from kinetic energy coupling and contains matrix elements $\mathcal{T}_{a b} \delta_{\alpha \beta}$. The two $5 \times 5$ matrix blocks in darker color contained in the main diagonal indicate inter-element points connecting contiguous elements (see text).

Restoring some flexibility in the choice of the basis used for the $\Omega$ part but still leaving quite a large sparsity in the full Hamiltonian matrix can also be obtained by imposing that the basis does not vary within each element. This means that the dependence of $\phi_{\alpha}^{(a)}$ on $(a)$ is replaced by an ensemble of basis functions in which the same basis is used for all points within the same element $m$ with the exception of the inter-element points for which alternative basis functions may be used. One such example would be the set of eigenvectors obtained through diagonalization of the reduced Hamiltonian $V\left(R_{x}, \Omega\right)$ at a fixed point $R_{x}$ inside the element (diabaticby-sector method ${ }^{21}$ ). The diabatic-by-sector approach trades loss of some sparsity with a (possible) reduction in the basis size.

As an example, the matrix structure for a more flexible basis choice is depicted in Fig. 2 in which different numbers of Lobatto points are used $P_{1}=4, P_{2}=5$, and $P_{3}=7$. In addition, the first element contains 5 basis functions, whereas the other two contain 4 alternative basis functions. The basis associated with the first inter-element point is taken to be identical to the 5 basis functions of the first element. The modification of the basis between elements 1 and 2 induces additional non-zero elements (pink in the figure) due to basis overlaps.

In the extreme case where the Hamiltonian is diagonalized at each point, one retrieves the slow-variable formulation proposed for hyperspherical bound states in Ref. 17. Such a fully adiabatic procedure does optimize the basis size but results in full overlap matrices at all off-diagonal grid elements, putting severe memory constraints on the size of treatable systems.

Realistic calculations usually require a large number of elements and points to be converged. Therefore, in the locally diabatic formulation, the empty part of the matrix becomes large and the filling factor decreases significantly. More

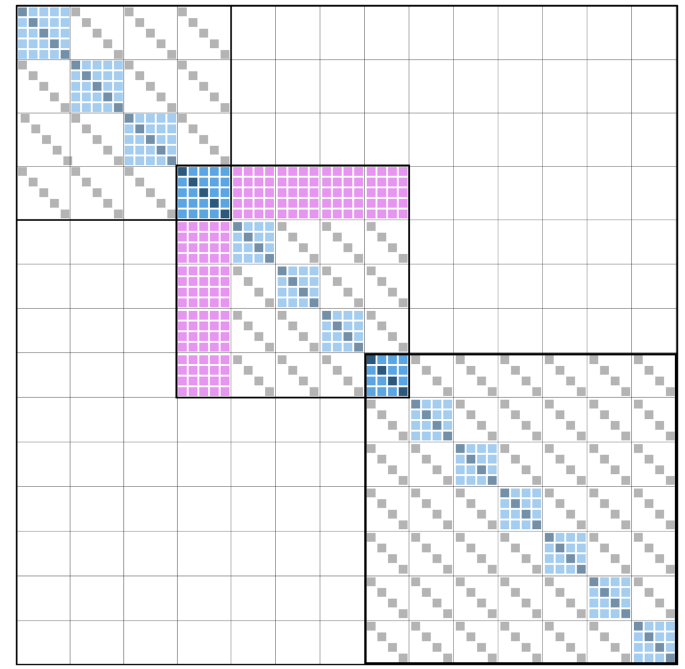

FIG. 2. Same as in Fig. 1 with 3 elements but now varying number of Lobatto points in each element (4, 5, and 6 , respectively) and different channel bases in elements 1 and 2. The overlap matrix in Eq. (18) is now a full matrix giving rise to the full off-diagonal blocks (pink) containing elements $\mathcal{T}_{a b} \mathcal{O}_{a \alpha, b \beta}$.

quantitatively, let us consider a potential represented by a full matrix with $N$ channels. For a partition composed of $M$ elements with $P$ points per element, the number of nonzero elements is $N(N+1)[M(P-1)+1] / 2+P(P-1) M N / 2$ that reduces to $\approx L N[N+P+1] / 2$ for $M, P \gg 1$. Note that due to matrix symmetry only elements above (or below) the main diagonal have been taken into account. The total number of elements (now both above and below the main diagonal) is $N^{2}[M(P-1)+1]^{2} \approx(N L P)^{2}$, resulting in a filling factor $\approx(N+P+1) /\left(2 N L P^{2}\right)$ that may easily drop below $1 \%$. We remark that this worst case scenario of a full potential matrix seldom happens in molecular physics due to the tensor nature of at least part of the interaction and to the accompanying selection rules.

Depending on the problem, purely diabatic and diabaticby-sector representation can also be conveniently combined. For instance, in Ref. 22 the present algorithm was used to join a purely diabatic spherical harmonics basis at a short range with a diabatic-by-sector representation at a long range to treat the difficult problem of ultracold collisions between two polar molecules in an optical tube.

\section{A. Bound states}

Bound states $\Psi^{(n)}$ of the system with energy $E_{n}$ are calculated imposing that the solution of the Schrödinger equation vanishes at the boundaries $R_{1}$ and $R_{L}$ of the radial interval. Accordingly, for $a=1$ and $L$, the expansion coefficients $F_{a \alpha}^{(n)}=0$, and all elements of the discretized Hamiltonian with grid indices $a, b=1, L$ in the system of Eq. (18) can be dropped.

The resulting equations for the multichannel bound-state solution at the remaining $L-2$ points present themselves in the form of a generalized eigenvalue problem

$$
\begin{gathered}
\sum_{b=2}^{L-1} \sum_{\beta=1}^{N^{(b)}} \mathcal{T}_{a b} \mathcal{O}_{a \alpha, b \beta} F_{b \beta}^{(n)}+\frac{2 \mu}{\hbar^{2}} \sum_{\beta=1}^{N^{(a)}} \omega_{a} \mathcal{U}_{\alpha \beta}\left(R_{a}\right) F_{a \beta}^{(n)} \\
=E_{n} \omega_{a} F_{a \alpha}^{(n)}, \quad a=2, \ldots, L-1 .
\end{gathered}
$$


The $\omega_{a}$ factor on the rhs can be removed by redefining as new unknown $\sqrt{\omega_{a}} F_{a \alpha}^{(n)}$ and by right multiplication by the diagonal matrix with element $1 / \sqrt{\omega_{a}}$. In this way, the problem is expressed as an ordinary eigenvalue problem. Finally note that, if needed, Eq. (18) with left grid index $a=1$ and $L$ can be used to compute the normal derivative components $\xi_{a \alpha}^{(n)}$ as a function of $F_{b \alpha}^{(n)}$ with $b=2, \ldots, L-1$.

\section{B. Scattering states}

In the case of scattering solutions, a number $N^{(L)}$ of linearly independent solutions with energy $E$ can be built from Eq. (18). We consider for definiteness the most common case where at the left end point $R_{1}$ the wavefunction vanishes. As in the bound-state problem, this implies that all lines and columns in the system of Eq. (18) with grid index $a=1$ can be dropped. At the other edge of the grid, we discuss below two approaches to obtain the physical wavefunction and thus the relevant scattering observables. The first one requires us to compute a matrix comprising all linearly independent solutions of the discretized Schrödinger equation, and the second one can be used to determine a single column-vector solution.

\section{R-matrix boundary conditions}

The so-called $R$-matrix solutions $\bar{\Psi}^{(\gamma)}$ are defined by the condition that their normal derivative vanishes on the surface $R=R_{L}$ in all but channel $\gamma$, where it is unity. Therefore, such $N^{(L)}$ independent $R$-matrix solutions with energy $E$ can be determined by imposing $\xi_{\alpha L}=\delta_{\alpha \gamma}$ and solving the linear system

$$
\begin{gathered}
\sum_{b=2}^{L} \sum_{\beta=1}^{N^{(b)}} \mathcal{T}_{a b} \mathcal{O}_{a \alpha, b \beta} \bar{F}_{b \beta}^{(\gamma)}+\frac{2 \mu}{\hbar^{2}} \sum_{\beta=1}^{N^{(a)}} \omega_{a}\left[\mathcal{U}_{\alpha \beta}\left(R_{a}\right)-E \delta_{\alpha \beta}\right] \bar{F}_{a \beta}^{(\gamma)} \\
=\delta_{L a} \delta_{\alpha \gamma}, \quad a=2, \ldots, L
\end{gathered}
$$

The $R$-matrix $\mathbf{R}$ is simply defined as the matrix with elements $R_{\alpha \beta}=\bar{F}_{L \alpha}^{(\beta)}$.

Solutions with physical boundary conditions can be written for $R \geq R_{L}$ as a superposition of solutions of the asymptotic Hamiltonian, comprising angular channel eigenfunctions $\Phi_{\alpha}^{(L+1)}$, and of reference regular $\tilde{f}$ and irregular $\tilde{g}$ radial functions,

$$
\Psi^{(I)}(R, \Omega)=\sum_{\alpha=1}^{N^{(L)}}\left[\tilde{f}_{\alpha}(R) \delta_{\alpha I}-\tilde{g}_{\alpha}(R) K_{\alpha I}\right] \Phi_{\alpha}^{(L+1)}(\Omega) .
$$

The channel eigenfunctions are $R$-independent and the superscript $(L+1)$ is merely introduced as an additional artificial grid point for ease of notation in subsequent formal manipulations. A solution $\Psi^{(I)}$ corresponds to a wave incoming in channel $I$ with scattered waves in all channels $\alpha$, with amplitudes $K_{\alpha I}$. If $f$ and $g$ are real standing waves, the coefficients $K_{\alpha I}$ form the reaction matrix $\mathbf{K}$.

The solutions $\Psi^{(I)}$ and its normal derivative can be expressed on the surface $R=R_{L}$ as linear combinations of the $R$-matrix solutions $\bar{\Psi}^{(\gamma)}$ with constant coefficients $N_{\gamma I}$,

$$
\Psi^{(I)}\left(R_{L}, \Omega\right)=\sum_{\gamma=1}^{N^{(L)}} \bar{\Psi}^{(\gamma)}\left(R_{L}, \Omega\right) N_{\gamma I}
$$

and

$$
\partial_{R} \Psi^{(I)}\left(R_{L}, \Omega\right)=\sum_{\gamma=1}^{N^{(L)}} \partial_{R} \bar{\Psi}^{(\gamma)}\left(R_{L}, \Omega\right) N_{\gamma I}
$$

Following the standard asymptotic matching procedure, ${ }^{21}$ Eq. (22) is inserted on the lhs of Eqs. (23) and (24) and the latter are projected on the angular basis $\Phi^{N^{(L)}}(\Omega)$. The resulting linear system can be easily solved for $\mathbf{K}$ in terms of $\mathbf{R}$,

$$
\mathbf{K}=\left(\mathbf{g}-\mathbf{R} \mathbf{g}^{\prime}\right)^{-1}\left(\mathbf{f}-\mathbf{R} \mathbf{f}^{\prime}\right) \text {. }
$$

Here, matrices $\mathbf{f}$ and $\mathbf{f}^{\prime}$ are, respectively, defined as

$$
f_{\alpha \beta}=\tilde{f}_{\alpha}\left(R_{L}\right) \mathcal{O}_{L \alpha,(L+1) \beta}, \quad f_{\alpha \beta}^{\prime}=\tilde{f}_{\alpha}^{\prime}\left(R_{L}\right) \mathcal{O}_{L \alpha,(L+1) \beta}
$$

as a function of the overlap between the asymptotic channels and the angular basis at the last grid point. A similar definition holds for $\mathbf{g}$ and $\mathbf{g}^{\prime}$.

\section{Scattering boundary conditions}

Rather than going through the determination of $N^{(L)}$ independent $R$-matrix solutions, scattering boundary conditions can also be incorporated directly in the linear system of Eq. (18). To this aim, we first impose that at the last grid point $a=L$, the wavefunction takes form (22),

$$
F_{L \alpha}^{(I)}=\sum_{\beta}\left[f_{\alpha \beta} \delta_{\beta I}-g_{\alpha \beta} K_{\beta I}\right] .
$$

Similarly, the normal derivative channel components on the rhs of Eq. (18) becomes

$$
\xi_{L \alpha}^{(I)}=\sum_{\beta}\left[f_{\alpha \beta}^{\prime} \delta_{\beta I}-g_{\alpha \beta}^{\prime} K_{\beta I}\right] .
$$

For notational ease, we define the matrix on the lhs of Eq. (18) as

$$
M_{a \alpha, b \beta}=\mathcal{T}_{a b} \mathcal{O}_{a \alpha, b \beta}+\frac{2 \mu}{\hbar^{2}} \omega_{a}\left[\mathcal{U}_{\alpha \beta}\left(R_{a}\right)-E \delta_{\alpha \beta}\right] \delta_{a b}
$$

As it will be clear from Eq. (30), in order to obtain a symmetric linear system, it is necessary to introduce the new unknown $\mathbf{X}=\mathbf{g K}$ in place of $\mathbf{K}$. We also define the logderivative ratio $\mathbf{Y}^{g}=\mathbf{g}^{\prime} \mathbf{g}^{-1}$, such that the quantity $\mathbf{g}^{\prime} \mathbf{K}$ on the rhs of Eq. (28) becomes $\mathbf{g}^{\prime} \mathbf{K}=\mathbf{Y}^{g} \mathbf{X}$.

With these definitions, the simple matrix algebra allows one to cast system (18) into the form

$$
\begin{gathered}
\sum_{b=2}^{L-1} \sum_{\beta=1}^{N^{(b)}} M_{a \alpha, b \beta} F_{b \beta}^{(I)}+\sum_{\beta=1}^{N^{(L)}}\left[M_{a \alpha, L \beta}+\delta_{L a} Y_{\alpha \beta}^{g}\right] X_{\beta}^{(I)} \\
=-\sum_{\beta=1}^{N^{(L)}} M_{a \alpha, L \beta} f_{\beta I}+\delta_{L a} f_{\alpha I}^{\prime} .
\end{gathered}
$$

As a final step, the $K$-matrix can be computed from the definition of $\mathbf{X}$ by solving the linear system $\mathbf{g K}=\mathbf{X}$. It is important to notice that for a given incoming wave labeled by index $I$, one can determine a single column of the matrix solution $\mathbf{X}$ and thus of $\mathbf{K}$. If one uses complex algebra and replaces $f_{\alpha}$ and $g_{\alpha}$ by traveling waves $h_{\alpha}^{(-)}$and $h_{\alpha}^{(+)}$, the asymptotic condition (22) becomes

$$
\Psi^{(I)}(R, \Omega)=\sum_{\alpha=1}^{N^{(L)}}\left[h_{\alpha}^{(-)}(R) \delta_{\alpha I}-h_{\alpha}^{(+)}(R) S_{\alpha I}\right] \Phi_{\alpha}^{(L+1)}(\Omega),
$$


with $\mathbf{S}$ the scattering matrix, whose elements are directly related to observables. The procedure to determine $\mathbf{K}$ presented in this section applies to the determination of $\mathbf{S}$, leading to the equivalent of Eq. (30) with $\mathbf{f}$ and $\mathbf{g}$ replaced by $\mathbf{h}^{(-)}$and $\mathbf{h}^{(+)}$, respectively, and $\mathbf{Y}^{g}$ by $\mathbf{Y}^{h^{(+)}}=\mathbf{h}^{(+)}\left[\mathbf{h}^{(+)}\right]^{-1}$. Determining a single column of interest of the scattering matrix may lead to computational advantages, in particular, in problems with a large number of open channels.

\section{Spectral log-derivative propagation}

In spite of the sparse character of the discretized Hamiltonian, memory can become a limiting factor for systems described by a large number of collision channels. In this case, it may be necessary to split the full propagation interval in smaller intervals, each comprising, for instance, only one element. The scattering equation is solved in any given element to determine at each point a matrix of linearly independent solutions $\mathbf{F}_{a}$ with elements $F_{\alpha a}^{(I)}$ labeled by column index $I$ and channel index $\alpha$. Such solutions will be combined to form the $\log$-derivative matrix $\mathbf{Y}_{a}=\mathbf{F}_{a}^{\prime} \mathbf{F}_{a}^{-1}$.

Our main equation (18) specialized to an element with $P_{m}$ points can now be rearranged as an algorithm expressing the value of $\mathbf{Y}_{P m}$ on the right-end of the element to a known input value $\mathbf{Y}_{1}$ assigned on the left-end point. This task can be accomplished by right multiplications by $\mathbf{F}_{P_{m}}^{-1}$ to give after simple algebra

$$
\begin{aligned}
& \sum_{b=1}^{P_{m}-1} \sum_{\beta=1}^{N^{(b)}}\left[M_{a \alpha, b \beta}+\delta_{1 a} Y_{1, \alpha \beta}\right] \bar{F}_{b, \beta \gamma}=-M_{a \alpha, P_{m} \gamma}, \\
& a=1, \ldots,\left(P_{m}-1\right) .
\end{aligned}
$$

With $\overline{\mathbf{F}}_{a} \equiv \mathbf{F}_{a} \mathbf{F}_{P_{m}}^{-1}$ determined at first $\left(P_{m}-1\right)$ points, the remaining equation at the last point

$$
Y_{P_{m}, \alpha \gamma}=\sum_{b=1}^{P_{m}-1} \sum_{\beta=1}^{N^{(b)}} M_{P_{m} \alpha, b \beta} \bar{F}_{b, \beta \gamma}+M_{P_{m} \alpha, P_{m} \gamma}
$$

determines the final log-derivative through a series of sparse matrix multiplications. The log-derivative $\mathbf{Y}_{P m}$ can then be used as an entry for the calculation in the next element.

\section{Error control}

A major advantage of the spectral element method is the possibility to estimate precisely the numerical error by a posteriori analysis of the calculated solution. To this aim, let us consider the solution wavefunction restricted to the $m$ th sector and suppose for the sake of simplicity that the angular basis of dimension $N_{m}$ is constant within the sector. The discretized solution at the $P_{m}$ points in the sector for the different channels is therefore represented by $P_{m} \times N_{m}$ elements noted as $F_{p \alpha}$.

The error is estimated by first performing an orthogonal transformation from the grid basis to the polynomial basis of Legendre polynomials $P_{n}(R)$ defined in the $\left[R_{1}^{(m)}, R_{P_{m}}^{(m)}\right]$ interval through a coordinate transformation in the same fashion as in Eq. (3). Assuming the polynomials normalized, the transformation matrix reads explicitly $O_{n p}=P_{n}\left(R_{p}\right) / \sqrt{w_{p}^{(m)}}$. For each channel $\alpha$, the transformation $\mathbf{O}$ gives the set of pseudospectral coefficients $\tilde{F}_{n \alpha}$ of the solution expanded on the
Legendre basis as $\tilde{F}_{n \alpha}=\sum_{n=1}^{P_{m}} O_{n p} F_{p \alpha}$. The main point is that the convergence of the Legendre polynomial series is superalgebraic, at least for sufficiently regular solutions. ${ }^{4}$ The size of last calculated coefficients $\tilde{F}_{P_{m} \alpha}$ is therefore a reliable estimate of the remainder of the series, i.e., of the numerical truncation error in each channel. If the error is larger (smaller) than a given tolerance criterion, one can either reduce (increase) the element size or increase (reduce) the polynomial order $P_{m}$. As recognized at the birth of the so-called $h p$-methods, the optimal strategy to guarantee an exponential accuracy of the calculated solution consists in increasing $P_{m}$ in the regions where the latter is regular and in decreasing the element size in the regions where it is irregular. ${ }^{23}$

We will show in Sec. III a series of numerical experiments for both scattering and bound state calculations. We limit ourselves to a relatively simple ro-vibrational model with a purely diabatic basis in order to make the numerical convergence analysis as plane as possible. Since as most usual in molecular physics the solution is regular, we fix the same polynomial order in all elements and study the behavior of selected observables as a function of both the element size and polynomial order.

\section{NUMERICAL TESTS}

We perform numerical tests of efficiency and accuracy of the algorithm on the $\mathrm{Rb}_{2} \mathrm{He}$ trimer, a system for which bound states and ultra-cold scattering properties have already been studied in our group. ${ }^{24,25}$

For the description of $\mathrm{Rb}_{2} \mathrm{He}$, the $\vec{R}$ and $\vec{r}$ Jacobi vectors are used. The corresponding Hamiltonian in the space fixed frame reads ${ }^{26}$

$$
\begin{aligned}
\hat{H}= & -\frac{\hbar^{2}}{2 \mu_{\mathrm{Rb}_{2}-\mathrm{He}}}\left(\frac{1}{R} \frac{\partial^{2}}{\partial R^{2}} R\right)-\frac{\hbar^{2}}{2 \mu_{\mathrm{Rb}_{2}}}\left(\frac{1}{r} \frac{\partial^{2}}{\partial r^{2}} r\right) \\
& +\frac{L^{2}}{2 \mu_{\mathrm{Rb}_{2}-\mathrm{He}^{2}} R^{2}}+\frac{j^{2}}{2 \mu_{\mathrm{Rb}_{2}} r^{2}}+\hat{V},
\end{aligned}
$$

where $R$ and $r$ are the $\mathrm{Rb}_{2}-\mathrm{He}$ and $\mathrm{Rb}_{2}$ distances, $\mu_{\mathrm{Rb}_{2}-\mathrm{He}}$ and $\mu_{\mathrm{Rb}_{2}}$ are the associated reduced masses, $\vec{L}$ and $\vec{j}$ are the angular momenta, and $\hat{V}$ is the potential term taken from Ref. $25 \mathrm{lim}-$ ited to the 2-body part. The generic $\Omega$ coordinates introduced in Sec. II correspond for this system to five spatial coordinates, namely, $r, \hat{r}$, and $\hat{R}$ that reduce to three when fixing the total angular momentum quantum numbers $J$ and $M$. The basis functions used to represent $\Phi_{a}(\Omega)$ in Eq. (17) are taken identical for all sectors. Equation (17) reads for this specific case

$$
\Phi_{a}(\Omega)=\sum_{v j \ell} v_{a, v j \ell} \frac{1}{r} \chi_{v j}(r) Y_{j \ell}^{J M}(\hat{r}, \hat{R}),
$$

where $\chi_{v j}(r)$ are the rovibrational eigenstates of the $\mathrm{Rb}_{2}$ diatomic and $Y_{j \ell}^{J M}$ are the coupled spherical harmonics. ${ }^{26}$

\section{A. Scattering states}

For the calculations, we vary $R$ from 4 to $120 a_{0}$, we use $\operatorname{Rb}_{2}(v=1, j=0)$ as the initial state for the collision, and we impose $R$-matrix boundary conditions. The linear system Eq. (21) is solved using the PARDISO package ${ }^{27,28}$ included in the MKL. ${ }^{29}$ This state-of-the art direct solver determines 
the solution of a sparse linear system in (number of nonzero elements) $)^{3 / 2}$ operations. In our tests, we find that the total memory used by PARDISO is about five times larger than the memory required to store the nonzero elements of the discretized Hamiltonian. The $K$-matrix, extracted from the matching procedure in Eq. (25) performed at $R=120 a_{0}$, is diagonalized to compute the eigenphase sum

$$
\delta=\sum_{i=1}^{n_{o p}} \arctan \left(\eta_{i}\right)
$$

with $\eta_{i}$ being the $n_{o p}$ eigenvalues of the open-open part of the $K$-matrix. Note that while the eigenphase sum is a function of collision energy and depends on the partial wave $J$ considered, the corresponding indices have been dropped for ease of notation.

For the accuracy tests, we used a collisional energy of $1 \mathrm{~K}$ above the $v=1, j=0$ initial state and focused on the $J=1$ partial wave. Basis functions with up to $v=4, j=24$ quantum numbers are included, resulting in 125 channels, 32 of which are energetically open at the considered collision energy. A fixed Lobatto order $P$ is used for all $M$ elements used for the discretization of the $\left[R_{1}, R_{L}\right]=[4,120]$ (in $a_{0}$ ) interval. All elements are taken of the same length, noted $h$ hereafter, with

$$
h=\left(R_{L}-R_{1}\right) / M .
$$

Figure 3 presents the variation of $\delta$ as a function of $h$ in the $\log -\log$ scale, when $h$ is systematically divided by two. Assuming a dependence of $\delta$ of the form

$$
\delta=\delta_{0}+C h^{\alpha},
$$

the power $\alpha$ is directly accessible by the slope of the variation of the quantity $\Delta \delta=\delta(h)-\delta(h / 2)$ as a function of $h$ in the $\log -\log$ scale, even when the exact value of $\delta_{0}$ is unknown. A comparison with the resolution of the coupled equations performed by the Johnson log-derivative propagator ${ }^{1}$ is also presented in the figure. The results for the Lobatto order $P=3$ case are strictly identical to the ones obtained with the

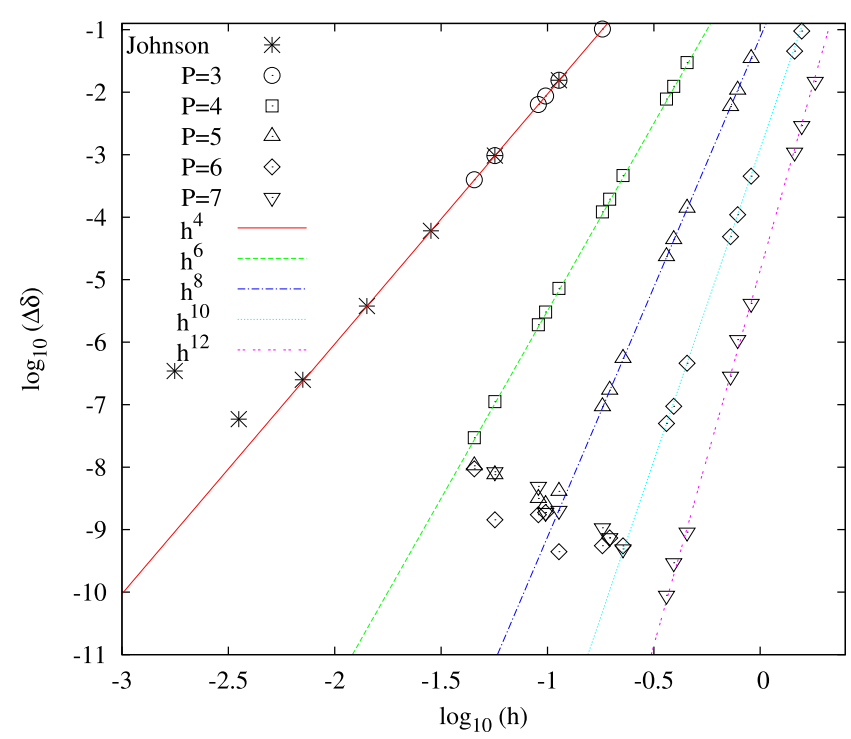

FIG. 3. Error as a function of element size for Johnson (star) and various Lobatto orders ( $\mathrm{P}=3$ to 7$)$. Relevant lines are also indicated.
Johnson propagator method. One can indeed show formally that the solution of Eq. (32) with two Lobatto points followed by the application of (33) gives exactly the same result as the three-point Johnson recursion. However, the two algorithms should not be viewed as equivalent, in the sense that the first half propagation step in Johnson's method is not equivalent to solving our Eq. (32) for two points. For these cases, the known $\alpha=4$ value can be read from the linear curves in the figure. For $\log _{10}(h)<-2.2$, the accuracy on $\delta$ can no longer be improved by a reduction of $h$ when using the Johnson propagator. For the $P=3$ Lobatto case, the memory requirement prohibits the computation at such small $h$. For the $P=4$ to $P=7$ Lobatto orders, a slope $\alpha=2 P-2$ is obtained. When increasing $P$, lower values of the absolute error are obtained for specific values of $h$. For example, a $10^{-10}$ accuracy is reached for $P=7$ and $h \sim 0.4 a_{0}$. We stress that $\delta$ is a very sensitive quantity and such an absolute error value on $\delta$ corresponds to the usually observed accuracy on rate coefficients calculations, much easier to converge.

An analysis of the central processing unit (CPU) time needed for given accuracies is presented in Table I. The table presents as a function of the Lobatto order $P$ the CPU in seconds needed to reach a given accuracy of $\log _{10}(\Delta \delta)$. The corresponding $h$ are also listed in the table. For these calculations, we increase $J$ to 10 for which the number of channels increases to 565 channels with 129 of them energetically open. The CPU given in the table corresponds to the resolution of the equations for one value of the collisional energy after an initialization step which is energy independent and thus to be performed only once if multiple collision energies are considered. The table shows that for a given accuracy, one gains in increasing the Lobatto order at least to the tested orders. The comparison of the CPU time needed by the Johnson ${ }^{1} \log$ derivative propagator is clearly in favor of this last approach when low accuracy is required. However, the situation changes when high accuracy is needed. In the present test, a $P=7$ computation is always more efficient than the Johnson version even for a single collision energy. When using the Gauss-Lobatto discretization, the CPU time requirement of the collisional energy dependent step is closely related to the number of integration points $L$ as underlined in Fig. 4. A roughly linear dependence of the CPU time as a function of the number of integration points is found for the Lobatto orders we tested. At a given number of integration points, the general trend is

TABLE I. CPU (in seconds) for various $P$ and $h$ combinations corresponding to two given values of the error on $\delta$ extracted from Fig. 3. The columns $E_{c o l}$

\begin{tabular}{|c|c|c|c|c|c|c|}
\hline \multirow[b]{2}{*}{ Type } & \multicolumn{3}{|c|}{$\log _{10}(\Delta \delta)=-2$} & \multicolumn{3}{|c|}{$\log _{10}(\Delta \delta)=-6$} \\
\hline & $h$ & $E_{c o l}$ ind. & $E_{c o l}$ dep. & $h$ & $E_{c o l}$ ind. & $E_{c o l}$ dep. \\
\hline$P=3$ & 0.10 & 368 & 2267 & & & \\
\hline$P=4$ & 0.38 & 140 & 795 & & & \\
\hline$P=5$ & 0.77 & 95 & 492 & 0.24 & 282 & 1556 \\
\hline$P=6$ & 1.23 & 73 & 373 & 0.49 & 180 & 814 \\
\hline$P=7$ & 1.81 & 59 & 339 & 0.80 & 141 & 778 \\
\hline Johnson & 0.10 & 354 & 80 & 0.01 & 3422 & 786 \\
\hline
\end{tabular}
ind. and $E_{c o l}$ dep. correspond to the CPU time of steps to be done once per collisional energy ( $E_{c o l}$ ind.) and at each collision energy ( $E_{c o l}$ dep.). 


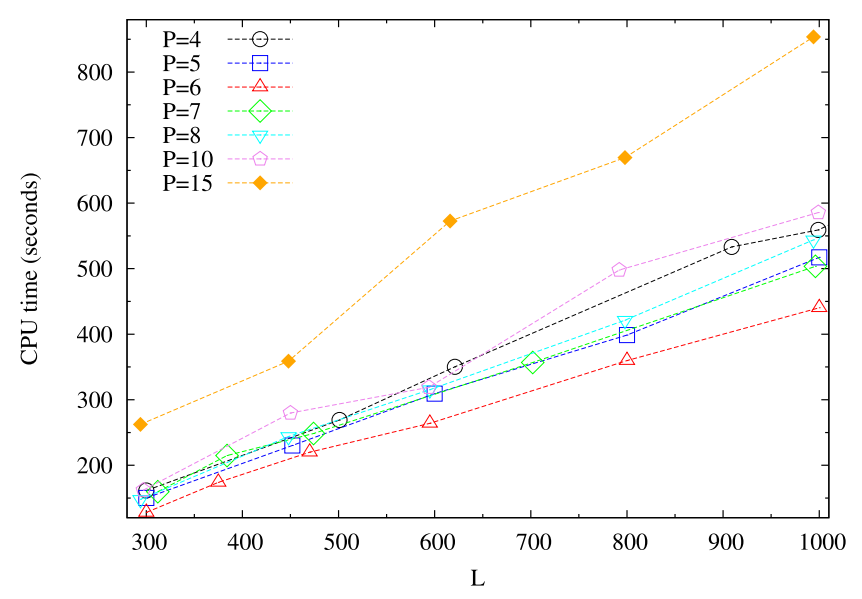

FIG. 4. CPU time in seconds for the energy-dependent part of the algorithm as a function of number of integration points $L$ for a selection of Lobatto orders, $P$ and $M$ elements. The data concern the $J=8$ partial wave computations.

an increase of the CPU time with the Lobatto order. Some exceptions are found like the $P=6$ case presented in Fig. 4 which turns out to be the cheapest calculation with respect to the CPU time for all numbers of integration points from 300 to 1000. We infer that this is due to particularities in the sparseness structure of the matrices handled by PARDISO. Similar but less marked exceptions have been found for the $J=10$ and $J=14$ partial wave computations.

\section{B. Bound states}

With the appropriate boundary conditions built in Eq. (20), bound states of the triatomic $\mathrm{Rb}_{2} \mathrm{He}$ can be determined using the same discretized Hamiltonian. We solve the sparse eigenvalue problem Eq. (20) using the density-matrix-based algorithm FEAST, a package included in MKL based on a contour representation of the resolvent in the complex plane. $^{30}$ Internally, FEAST solves a series of sparse linear systems using a user-defined subroutine, PARDISO in our case.

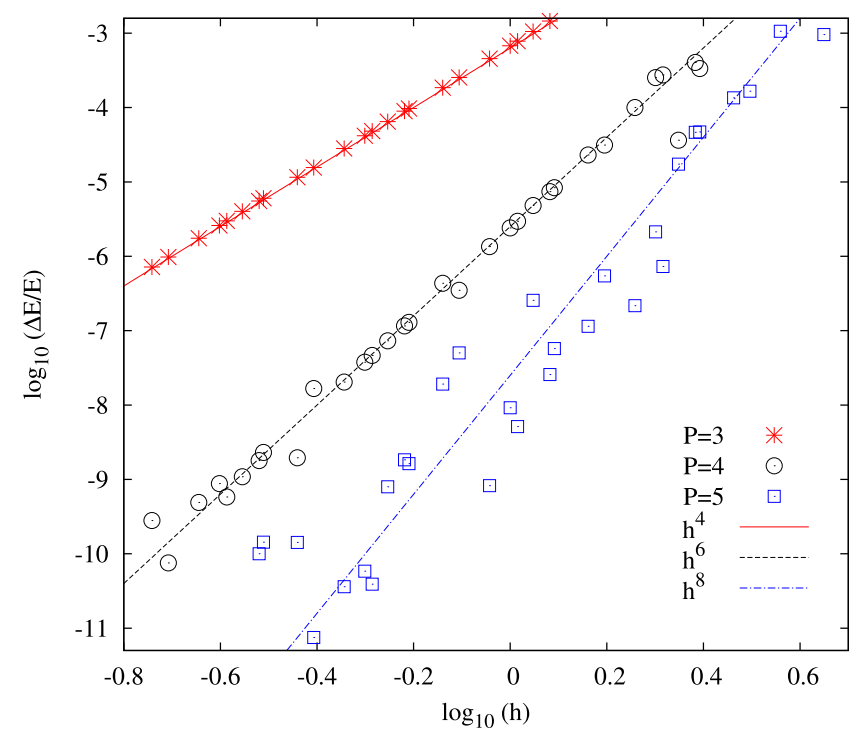

FIG. 5. Error as a function of element size for various Lobatto orders $(P=3$, 4 , and 5) together with relevant power lines in the log-log scale.
We focus on the $J=2$ partial wave of total parity $\Pi=-$ for which a single bound state is found. This state is weakly bound with respect to the $\mathrm{Rb}_{2}+\mathrm{He}$ asymptote, and an enlarged $R$ box with $R \in[4,236] a_{0}$ is used. The converged energy is -12.63 $\mathrm{mK}$ below the $\mathrm{Rb}_{2}+\mathrm{He}$ asymptote. Figure 5 presents the evolution of the relative error on the computed energy when increasing by two the element size $h$ for various Lobatto orders $P$ in the $\log -\log$ scale. For the lowest $P$ orders presented, the $h^{2 P-2}$ behavior is retrieved. For $P=5$, numerical noise increases when reducing $h$. This is partly due to the FEAST algorithm which implies an iterative procedure with two kinds of internal convergence criteria. One criterion tests the evolution of the energies from one iteration to the next one, and the second one is a maximum number of iterations. The data presented have been obtained with a $10^{-14}$ value and 50 iterations maximum for these two FEAST parameters.

\section{CONCLUSIONS}

In summary, we have explored the numerical performance of the spectral-element method in multichannel quantum dynamics. Combination of the spectral-element discretization with purely diabatic or diabatic-by-sector bases leads to a highly sparse representation of the Hamiltonian. This results in significant memory saving for the bound state problem as compared, for instance, to the scaled DVR approach. ${ }^{6,7}$ Regarding the scattering problem, accuracy is significantly less limited by round-off errors in the spectral element approach than in popular propagation methods, and the corresponding computation time is advantageous when the required accuracy is high.

In perspective, it may be interesting to test iterative rather than direct algorithms to solve the scattering linear system for the discretized Schrödinger equation, in particular, when boundary conditions of Eq. (30) are imposed to obtain a single column of the scattering matrix. In this case, if iterative solvers turned out to perform better than (number of nonzero elements) ${ }^{3 / 2}$, one might be able to overcome the (number of channel $)^{3}$ unfavorable computational cost scaling presented by time-independent calculations as compared to time-dependent calculations.

\section{ACKNOWLEDGMENTS}

This work is supported by the Agence Nationale de la Recherche (Contract COLORI No. ANR-12-BS04-0020-01).

${ }^{1}$ B. R. Johnson, J. Comput. Phys. 13, 445 (1973)

${ }^{2}$ D. E. Manolopoulos, J. Chem. Phys. 85, 6425 (1986).

${ }^{3}$ B. R. Johnson, J. Chem. Phys. 69, 4678 (1978).

${ }^{4}$ J. P. Boyd, Chebyshev and Fourier Spectral Methods (Dover, New York, 2001).

${ }^{5}$ J. V. Lill, G. A. Parker, and J. C. Light, Chem. Phys. Lett. 89, 483 (1982).

${ }^{6}$ E. Tiesinga, C. J. Williams, and P. S. Julienne, Phys. Rev. A 57, 4257 (1998).

${ }^{7}$ V. Kokoouline, O. Dulieu, R. Kosloff, and F. Masnou-Seeuws, J. Chem. Phys. 110, 9865 (1999).

${ }^{8}$ T. J. R. Hughes, The Finite Element Method: Linear Static and Dynamic Finite Element Analysis (Dover Publications, 2000).

${ }^{9}$ S. A. Orszag, J. Comput. Phys. 37, 70 (1980).

${ }^{10}$ G. E. Karniadakis and S. Sherwin, Spectral/hp Element Method for Computational Fluid Dynamics (Oxford University Press, 2005).

${ }^{11}$ C. Canuto, M. Hussaini, A. Quarteroni, and T. Zang, Spectral Methods: Evolution to Complex Geometries and Applications to Fluid Dynamics, Scientific Computation (Springer, Berlin, Heidelberg, 2007). 
${ }^{12}$ D. E. Manolopoulos and R. E. Wyatt, Chem. Phys. Lett. 152, 23 (1988).

${ }^{13}$ T. N. Rescigno and C. W. McCurdy, Phys. Rev. A 62, 032706 (2000).

${ }^{14}$ L. Tao, T. N. Rescigno, and C. W. McCurdy, Phys. Rev. A 79, 012719 (2009).

15 A. Scrinzi, Phys. Rev. A 81, 053845 (2010).

${ }^{16}$ O. I. Tolstikhin, S. Watanabe, and M. Matsuzawa, J. Phys. B: At., Mol. Opt. Phys. 29, L389 (1996).

${ }^{17}$ H. Suno, J. Chem. Phys. 134, 064318 (2011).

${ }^{18}$ B. I. Schneider, L. A. Collins, and S. X. Hu, Phys. Rev. E 73, 036708 (2006).

${ }^{19}$ D. A. Telnov and S.-I. Chu, Phys. Rev. A 76, 043412 (2007).

${ }^{20}$ S. X. Hu, Phys. Rev. E 81, 056705 (2010).

${ }^{21}$ J.-M. Launay and M. Le Dourneuf, Chem. Phys. Lett. 163, 178 (1989).
${ }^{22}$ A. Simoni, S. Srinivasan, J.-M. Launay, K. Jachymski, Z. Idziaszek, and P. S. Julienne, New J. Phys. 17, 013020 (2015).

${ }^{23}$ I. Babuška and H. C. Guo, Adv. Eng. Software 15, 159 (1992).

${ }^{24}$ G. Guillon, A. Viel, and J.-M. Launay, J. Chem. Phys. 136, 174307 (2012).

${ }^{25}$ A. Viel and J.-M. Launay, J. Phys. Chem. A 118, 6529 (2014).

${ }^{26}$ A. M. Arthurs and A. Dalgarno, Proc. R. Soc. A 256, 540 (1960).

${ }^{27}$ C. G. Petra, O. Schenk, M. Lubin, and K. Gärtner, SIAM J. Sci. Comput. 36, C139 (2014).

${ }^{28}$ C. G. Petra, O. Schenk, and M. Anitescu, Comput. Sci. Eng. 16, 32 (2014). ${ }^{29} \mathrm{Seehttps} / / /$ software.intel.com/en-us/forums/intel-math-kernel-library/topic /517994 for Intel(R) Math Kernel Library, version 11.2.2 for Linux, 2015.

${ }^{30}$ E. Polizzi, Phys. Rev. B 79, 115112 (2009). 